\title{
Tau drives translational selectivity by interacting with ribosomal proteins
}

\author{
Shon A. Koren ${ }^{1}$. Matthew J. Hamm ${ }^{1}$. Shelby E. Meier ${ }^{2}$ - Blaine E. Weiss ${ }^{2} \cdot$ Grant K. Nation $^{2} \cdot$ Emad A. Chishti $^{2}$.

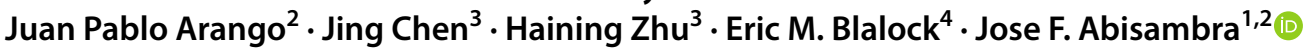

Received: 15 October 2018 / Revised: 31 January 2019 / Accepted: 1 February 2019 / Published online: 13 February 2019

(C) The Author(s) 2019

\begin{abstract}
There is a fundamental gap in understanding the consequences of tau-ribosome interactions. Tau oligomers and filaments hinder protein synthesis in vitro, and they associate strongly with ribosomes in vivo. Here, we investigated the consequences of tau interactions with ribosomes in transgenic mice, in cells, and in human brain tissues to identify tau as a direct modulator of ribosomal selectivity. First, we performed microarrays and nascent proteomics to measure changes in protein synthesis. Using regulatable $\mathrm{rTg} 4510$ tau transgenic mice, we determined that tau expression differentially shifts both the transcriptome and the nascent proteome, and that the synthesis of ribosomal proteins is reversibly dependent on tau levels. We further extended these results to human brains and found that tau pathologically interacts with ribosomal protein S6 (rpS6 or S6), a crucial regulator of translation. Consequently, protein synthesis under translational control of rpS6 was reduced under tauopathic conditions in Alzheimer's disease brains. Our data establish tau as a driver of RNA translation selectivity. Moreover, since regulation of protein synthesis is critical for learning and memory, aberrant tau-ribosome interactions in disease could explain the linkage between tauopathies and cognitive impairment.
\end{abstract}

Keywords Tau $\cdot$ Transcriptome $\cdot$ Translation $\cdot$ Nascent proteomics $\cdot$ Ribosome $\cdot$ rpS6

\section{Introduction}

Neurons require constant protein production for synaptic function and are, therefore, particularly vulnerable to chronic attenuation of protein synthesis [35]. Transient

Electronic supplementary material The online version of this article (https://doi.org/10.1007/s00401-019-01970-9) contains supplementary material, which is available to authorized users.

Jose F. Abisambra

j.abisambra@ufl.edu; jose.abisambra@gmail.com

1 Department of Neuroscience and Center for Translational Research in Neurodegenerative Disease, University of Florida, 1275 Center Drive, BOX 100159, Gainesville, FL 32610, USA

2 Sanders Brown Center on Aging, Department of Physiology, Spinal Cord and Brain Injury Research Center, and Epilepsy Center, University of Kentucky, Lexington, KY 40513, USA

3 Department of Molecular and Cellular Biochemistry, University of Kentucky, Lexington, KY 40513, USA

4 Department of Pharmacology and Nutritional Sciences, University of Kentucky, Lexington, KY 40513, USA suppression of translation is a cellular strategy to overcome conditions such as endoplasmic reticulum stress [20]. However, chronic suppression of protein synthesis contributes to the pathogenesis of multiple neurodegenerative disorders including tauopathies [4]. Pronounced ribosomal deficiencies appear in regions where tau pathology is evident, yet the link between tau and ribosomal function has not been established [14]. Furthermore, memory formation requires protein synthesis [15, 22]. Since progressive memory loss is a common and early symptom of virtually all tauopathies, and the processes of learning and memory are intricately dependent on de novo protein synthesis, ribosomal dysfunction could be an underlying mechanism driving these disorders.

Tau normally binds to ribosomes in the brain, and this interaction is enhanced in tauopathies [34]. In fact, hyperphosphorylated tau complexes with ribosomes in early stages of pathological tau aggregation $[25,38,39,42-44$, 57], and tauopathic brains have reduced ribosomal function $[14,28,32,50]$. These data suggest that alterations to the tau-ribosome complex could be an early pathogenic event in these disorders. 
Our recent studies show that ribosomes associate with both pathological and non-pathological tau [35, 36]. Yet, the consequences of these interactions are still unknown. The emerging concept of ribosome specialization, where accessory proteins promote ribosomal selectivity for translation of distinct mRNAs, suggests an entirely new mechanism for regulation of protein synthesis $[8,53,54,61]$. Considering that tau associates with ribosomal proteins [19] and that pathological tau modifies the rate of translation [34], we hypothesized that tau alters ribosome function thereby promoting translation of distinct transcripts.

To test this hypothesis, we used several in vivo and in vitro models, as well as human Alzheimer's tissue, where disease-associated tau species are enriched. We show that tau expression impairs protein translation by measuring protein synthesis in vivo in the brain with puromycin labeling of nascent peptides. Then, using a transcriptomics-toproteomics approach, we identified a tau-driven disparity between gene transcription and protein synthesis. Interestingly, tau decreased protein synthesis of ribosomal genes but not their transcription in tau transgenic mice. We hypothesized this unequal distribution was based on tau altering the function of ribosomal protein S6 (rpS6 or S6), which is involved in the regulation of ribosomal protein synthesis $[18,62]$. We also found that tau interferes with S6 activation, and this interaction correlates with the decreased translation of transcripts coding for ribosomal proteins in Alzheimer's disease (AD) brains. Consistent with our previous findings, these new data suggest the overall loss of translation found in tauopathies may be the result of a pathological gain-offunction of tau, where it attenuates translation by reducing the function or availability of S6.

\section{Methods}

\section{Mice}

The Institutional Animal Care and Use Committee (IACUC) of the University of Kentucky approved the use of animals in this study, which were conducted in accordance with the principles of animal care and experimentation in the Guide For the Care and Use of Laboratory Animals. Parental rTg4510 ( Tg) mice were obtained from The Jackson Laboratories (stock \#024854) and backcrossed for at least five generations onto FVB/NJ non-transgenic (Non) mice (stock \#001800) and genotyped as described previously [51]. Tg mice and Non mice (littermate controls) were housed in a $14 \mathrm{~h} \mathrm{light} / 10 \mathrm{~h}$ dark cycle at a constant temperature $\left(23{ }^{\circ} \mathrm{C} \pm 2{ }^{\circ} \mathrm{C}\right.$ ) with food and water available ad libitum. Doxycycline treatment consisted of feeding mice a doxycycline diet (200 ppm, Envigo TD.00502) for 35 days with animals killed on the final day of treatment [4]. Tg and Non mice used for puromycin immunostaining were gavaged once a day for 30 days with $0.5 \%$ hydroxypropyl methylcellulose $+0.1 \%$ Tween- 80 in water at $\mathrm{pH} 4$.

\section{Human brain samples}

Human samples were obtained from the University of Kentucky (UK) Alzheimer's Disease Center. Sample collection and experimental procedures involving human tissue were in compliance with the UK Institutional Review board. Samples from Brodmann areas 21/22 (superior temporal gyrus) were used. Patient demographics are included as a table in Online Resource 1.

\section{In vivo puromycin administration}

Mice were injected intraperitoneally with $225 \mathrm{mg} / \mathrm{kg}$ puromycin suspended in water (Research Products International, P33020). After $25 \mathrm{~min}$, they were placed in an isoflurane anesthesia chamber for $5 \mathrm{~min}$. Approximately $5 \mathrm{~min}$ following isoflurane exposure, animals were transcardially perfused for 5-10 min post injection with $0.9 \%$ saline. Brains were immediately harvested and the hemispheres anatomically separated and snap frozen in liquid nitrogen for downstream processing or drop-fixed in $4 \%$ para-formaldehyde for immunohistochemical studies. Brain laterality was maintained throughout experiments.

\section{Puromycin immunohistochemistry}

Immunohistochemistry was performed as described previously [2]. Briefly, 4\% para-formaldehyde drop-fixed brain samples were cryoprotected by incubating in sequential concentrations of sucrose $(10 \%, 20 \%$, and $30 \%)$ sucrose for $24 \mathrm{~h}$ each. Samples were frozen on a temperature-controlled freezing stage, sectioned $(25 \mu \mathrm{m})$ on a sliding microtome, and stored in a solution of PBS containing $0.02 \%$ sodium azide at $4{ }^{\circ} \mathrm{C}$. Free-floating tissue was treated with $3 \%(\mathrm{v} / \mathrm{v})$ hydrogen peroxide $+10 \%(\mathrm{v} / \mathrm{v})$ methanol in Tris-buffered saline (TBS, pH 7.4) to quench endogenous peroxidase activity. The Mouse on Mouse (MOM) Detection Kit (Vector Labs, BMK-2202) was used for blocking and staining procedures, with buffers prepared as described in standard protocol supplied with the kit. Sections were then incubated in Mouse Ig blocking buffer for $1 \mathrm{~h}$ at RT. Sections were incubated overnight at $4{ }^{\circ} \mathrm{C}$ with puromycin monoclonal antibody at 1:100 (EMD Millipore, MABE343) in MOM Diluent. Sections were washed with TBS and incubated with biotinylated anti-mouse IgG (Vector Laboratories) for $10 \mathrm{~min}$ at RT. Sections were washed again and incubated in ABC solution (Vector Laboratories) for $10 \mathrm{~min}$ at RT. Sections were washed again and incubated in diaminobenzidine (Sigma-Aldrich) and hydrogen peroxide in TBS. Sections 
were washed, mounted, and cover-slipped using Depex mounting media (Electron Microscopy Science). Images of the cortex (specifically somatosensory cortex, SSp) and hippocampus (CA1) were taken and quantified together for analysis of regions with severe tau pathology in $\mathrm{rTg} 4510$ mice. All values were normalized to signal in non-transgenic control mice.

\section{Sample tissue homogenization}

Brain samples from human patients $(\sim 100 \mathrm{mg})$ or from mice $(\sim 50 \mathrm{mg})$ were mechanically homogenized in RIPA lysis buffer (Thermo 89900) with protease inhibitors (Sigma 4693159001), PMSF (1 mM final concentration), and phosphatase inhibitors (Gibco 786-452 and -451) as previously described [5]. Samples were centrifuged at $4{ }^{\circ} \mathrm{C}$ at $13,000 \times g$ for 15-25 min, and the supernatant was used for subsequent steps. Protein concentrations were quantified using the Pierce BCA kit (Thermo Fisher, 23225).

\section{Western blotting}

Western blot experiments were performed as described previously [26]. Sample lysate protein concentrations were normalized with lysis buffer and denatured with $4 \times$ Laemmli buffer with $10 \% \beta$-mercaptoethanol. Proteins were resolved in $10 \%$ Tris-Glycine gels (BioRad) and transferred onto polyvinylidene fluoride (PVDF) membranes (Millipore, IPVH00010). Membranes were blocked in 1X PBS with $0.1 \%$ Tween-20 (PBS-T). All antibodies were diluted in 5\% milk or 5\% BSA in PBS-T. Primary antibodies were used as follows: PHF1 (1:2000, generously provided by Dr. Peter Davies), H150 total tau (1:2000, SantaCruz), Tau 5 total tau (1:2000, Millipore), actin (1:5000, Cell Signaling Technology), GAPDH (1:5000, Cell Signaling Technology), RPL28 (1:1000, GeneTex), EIF3E (1:1000, Sigma-Aldrich), Phospho-RPS6 Ser240/244 (1:1000, Cell Signaling Technology), total RPS6 (1:1000, SantaCruz). Bands were detected using ECL (GE Amersham Imager 600) using SuperSignal West Pico (Thermo Fisher, 1863096). Blot images were quantified using ImageJ (1.52b) and normalized to either GAPDH or $\beta$-actin.

\section{Puromycin immunoprecipitation}

Exactly $400 \mu \mathrm{g}$ of protein were brought to $500 \mu \mathrm{l}$ with Hsiao-TBS and incubated with $5 \mu$ of anti-puromycin antibody (Millipore, mabe434) overnight at $4^{\circ} \mathrm{C}$ under rotation. Approximately $150 \mu \mathrm{g}$ Protein G Dynabeads (Thermo Fisher, 10003D) were resuspended in $50 \mu 10 \mathrm{mM}$ Tris $(\mathrm{pH}$ 7.5) and crosslinked with BS3 and then incubated with the sample-antibody complex for 2-3 h at RT under rotation. Beads were washed twice with washing buffer $(10 \mathrm{mM}$ Tris,
$50 \mathrm{mM} \mathrm{NaCl}, \mathrm{pH} 7.5$ ) containing $0.2 \%$ Tween-20 and twice without Tween-20. Samples were eluted with $25 \mu \mathrm{l}$ containing $100 \mathrm{mM}$ glycine ( $\mathrm{pH}$ 3.0) for $10 \mathrm{~min}$ at RT, and subsequently quenched with equal volume of $10 \mathrm{mM}$ Tris $(\mathrm{pH}$ 8.0). Eluted sample protein concentrations were quantified at approximately $10 \mu \mathrm{g}$.

\section{Nascent protein proteomics}

Proteins eluted from the puromycin immunoprecipitation were run via SDS-PAGE. Each lane in the gel was excised into 12 major portions and subjected to dithiothreitol reduction, iodoacetamide alkylation, and in-gel trypsin digestion using a standard protocol as previously reported $[13,63]$. The resulting tryptic peptides were extracted, concentrated to $15 \mu \mathrm{l}$ using a SpeedVac, and $5 \mu \mathrm{l}$ were injected for nanoLC-MS/MS analysis [33]. LC-MS/MS data were acquired on an LTQ Velos Orbitrap mass spectrometer (Thermo Fisher Scientific, Waltham, MA) coupled to a Nano-LC Ultra/cHiPLC-nanoflex HPLC system (Eksigent, Dublin, CA) through a nano-electrospray ionization source. The tryptic peptide sample was injected with an autosampler, desalted on a trap column, and subsequently separated by reverse phase $\mathrm{C} 18$ column ( $75 \mathrm{~mm}$ i.d. $\times 150 \mathrm{~mm}$ ) at a flow rate of $250 \mathrm{~nL} / \mathrm{min}$. The HPLC gradient was linear from 5 to $60 \%$ mobile phase B for 30 min using mobile phase A $\left(\mathrm{H}_{2} \mathrm{O}, 0.1 \%\right.$ formic acid) and mobile B (90\% acetonitrile, $0.1 \%$ formic acid). Eluted peptides were analyzed using data-dependent acquisition: peptide mass spectrometry data were obtained by Orbitrap with a resolution of 60,000 . The seven most abundant peptides were subjected to collision-induced dissociation and MS/MS analysis in LTQ linear trap. The LC-MS/MS data were submitted to a local MASCOT server for MS/MS protein identification search via the ProteomeDiscoverer software. The mass error tolerance was $5 \mathrm{ppm}$ for peptide MS and 0.8 Da for MS/ MS. All peptides were required to have an ion score greater than $30(p<0.05)$. The false discovery rate in each LC-MS/ MS analysis was set to be less than $1 \%$. Two samples, one non-transgenic and one rTg4510, were immunoprecipitated with IgG antibody and analyzed via LC-MS/MS to determine inherent non-specific binding. Any matching proteins from these samples were removed from all samples prior to analysis. Only proteins with one or greater unique peptides were considered in the analysis. For pathway analysis, proteins which passed all filtering criteria were analyzed in the Database for Annotation, Visualization and Integrated Discovery (DAVID) [23] against the mouse genome. Only processes with an adjusted $p$ value less than 0.1 were considered significant and selected for downstream analysis and are reported in Fig. 3. Since no unique annotations were reported between Non + Veh and Non + Doxycycline groups, the lists of proteins were combined to form the Non-group in 
analysis. Comparative ontology analysis was done by grouping significant annotation terms according to six distinct groups related to brain function. For each group, the number of terms were compared as a ratio to the number found in non-transgenic controls for the same class. Annotations were functionally grouped and presented as a ratio of the number of annotations assigned to each group relative to the number in Non + Veh. All proteins, brain-related annotation terms, and comparative analyses and grouping are reported in Online Resource 2.

\section{Microarray and transcript post-hoc template matching}

Isolated RNA (100 $\mu \mathrm{g}$ per sample) was loaded onto a 96-well plate and shipped to Thermofisher (San Diego, CA) for array processing. High quality extracted RNA (RIN > 8.9) was labeled and hybridized to Mouse ClariomD microarrays (Clariom, ThermoFisher). One sample in the rTg4510 + normal feed ( $\mathrm{Tg}+\mathrm{Veh}$ ) group did not pass quality control (PCA analysis and Pearson correlation matrix) and was removed from subsequent analyses. Signal intensities were calculated using the Robust Multi-Array Algorithm [11] and are reported on the $\log 2$ scale. Transcript clusters were annotated to gene symbols using NetAffx annotation files (Release 36). The full transcriptional profile data set is available through the Gene Expression Omnibus under accession ID: GSE121264. For the purposes of this analysis, pre-statistical filtering was performed as in prior work $[6,16,30]$, and included retaining uniquely annotated transcript clusters with reliable signal strength (RMA signal $>6.76$ on at least 1 array). Intensity values of these pre-statistically filtered genes were then analyzed to identify differentially expressed genes (DEGs) with twoway ANOVA $(p \leq 0.01)$. For ANOVA analysis, the false discovery rate (FDR) estimate of multiple testing error is reported in Results. Although the ANOVA test identifies DEGs, it does not determine patterns of expression among those DEGs. To do this, DEGs were analyzed post hoc using a template matching strategy as in previous work $[10,16$, 27]. Briefly, group mean intensities for each DEG were correlated with idealized templates representing six canonical expression patterns: rescued by tau reduction, resistant to tau suppression, altered by doxycycline treatment, altered by doxycycline and transgenic tau expression, altered by doxycycline only in non-transgenics, and altered by doxycycline only in transgenics. For example, the ideal template for "rescued by tau reduction" is represented by $(0,0,1,0)$ for Non + Veh, Non + Dox, Tg + Veh, and Tg + Dox groups. The mean intensities $(\log 2)$ for example transcript Lyz1 are $(5.64,6.65,7.29,6.03)$, resulting in a correlation of $r=0.97$ to this template. Finally, the sign of the correlation indicates whether the transcript matched the pattern (positive, e.g., upregulated) or matched the mirror-reflection of the pattern (negative, e.g., downregulated). DEGs were assigned to the template of highest correlation if Pearson's $r \geq|0.85|$. To further refine analysis, templates assigned significantly more genes than expected by chance (binomial test, $p \leq 0.05$ ) were considered enriched and used for subsequent analysis. All DEGs and transcripts attributed to a pattern are listed in Online Resource 3.

\section{RNA isolation and quantitative real-time PCR}

RNA was isolated from $\sim 100 \mathrm{mg}$ of human brain or $\sim 50 \mathrm{mg}$ of frozen cortex of rTg4510 and littermate control mice as described in [3] using TRIzol reagent (Ambion) with Proteinase K digestion (ThermoFisher, EO0491), then column purified using PureLink (ThermoFisher). RNA was measured for integrity on an Agilent 2100 Bioanalyzer and only samples $>8.9$ RNA integrity number (RIN) were used in downstream analysis. RIN did not significantly differ between groups $(p>0.7)$. For qRT-PCR, gene transcription was evaluated by TaqMan probes and intensities were normalized to GAPDH expression as an internal control. Fold change was determined using the $2^{-\Delta \Delta C t}$ method. Kruskal-Wallis and Dunn's test $p$ values are presented in Online Resource 4.

\section{Cell culture}

We cultured tetracycline-inducible HEK cells which express wild-type human 0N4R tau, termed iHEK Tau cells, as previously described [4]. To induce tau expression, cells were treated with tetracycline $(1 \mu \mathrm{g} / \mathrm{ml}$; Sigma) for either 24 or $96 \mathrm{~h}(\mathrm{ON})$ and either immediately harvested or harvested following $24 \mathrm{~h}$ tetracycline washout (OFF) with fresh media. $60 \mathrm{~min}$ before harvesting across all groups, puromycin was added to the media to a final concentration of $10 \mu \mathrm{g} / \mathrm{ml}$. Next, cells were washed $2 \times$ with ice-cold PBS and lysed using RIPA buffer (Thermo 89900) with protease and phosphatase inhibitors as previously described [34]. Protein concentrations were quantified using the Pierce BCA kit (Thermo Fisher, 23225).

\section{Results}

We recently used in vitro models to demonstrate that misfolded, oligomeric, and hyperphosphorylated tau species reduce the rate of translation [34]. However, whether tau impairs translation in the brain remains unknown. Therefore, we measured changes in RNA translation using a puromycin-based assay (Surface Sensing of Translation or SUnSET) adapted for use in vivo [52]. As a structural analog of tRNA, puromycin is stably incorporated into growing 
polypeptide chains and these newly synthesized proteins can be detected with anti-puromycin antibodies. We performed SUnSET in $5 \mathrm{mo}$ and $7 \mathrm{mo}$ rTg 4510 tau transgenic (Tg) mice to investigate whether tau alters protein synthesis as pathology increases. At $5 \mathrm{mo}, \mathrm{Tg}$ mice have robust expression of a disease-associated human mutant tau (P301L), tau deposition into tangles, mild cognitive deficits, altered neuronal plasticity, and moderate brain atrophy. At $7 \mathrm{mo}$, Tg mice have severe morphological and cognitive damage as well as extensive neuronal death and reduced protein synthesis $[2,47,48,51]$.

We found that despite an appreciable but non-significant reduction at $5 \mathrm{mo}(\sim 34 \%, p=0.06)$, puromycinylated protein signal was significantly reduced in $7 \mathrm{mo}$ Tg mice compared to non-transgenic (Non) littermate controls in regions with strong tau expression (Fig. 1a-e). Interestingly, $7 \mathrm{mo} \mathrm{Tg} \mathrm{mice}$ also neared a significant reduction compared to $5 \mathrm{mo} \mathrm{Tg}$ $(\sim 31 \%, p=0.071)$. Global translation in the brain of $\mathrm{Tg}$ mice is impaired at $7 \mathrm{mo}$ but not at $4 \mathrm{mo}$ due to activation of the unfolded protein response mediated by the ER stress protein PERK [47]. To validate this previous report, we immunoblotted for p-eIF $2 \alpha$ and total eIF $2 \alpha$ levels in non-transgenic and $\mathrm{Tg}$ mice treated with and without a PERK inhibitor and detected no activation of the PERK pathway of the UPR
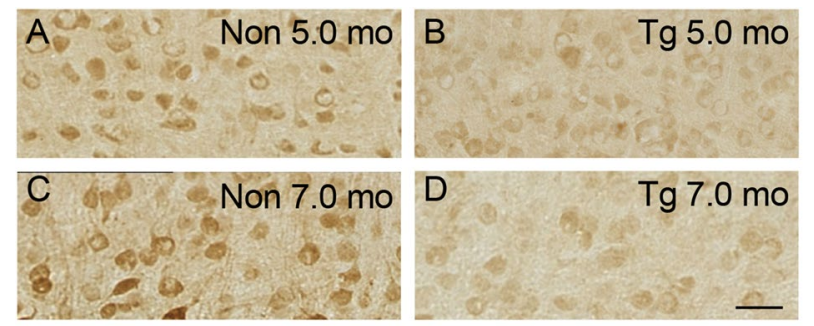

E

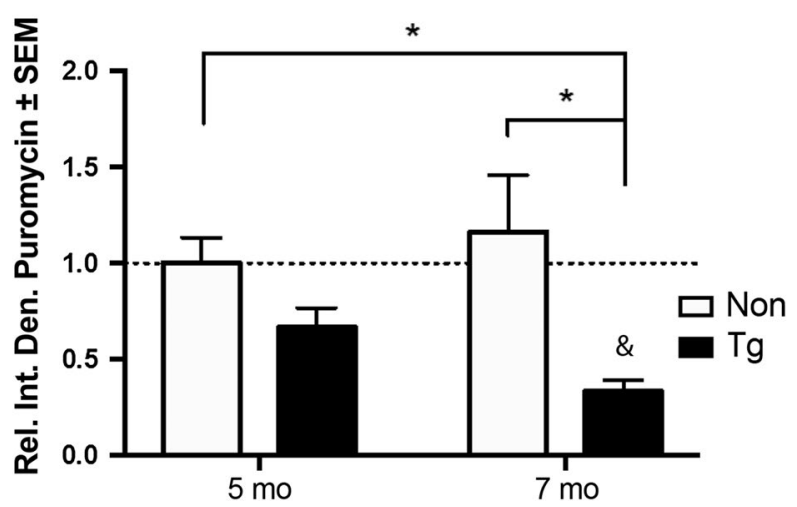

Fig. 1 Protein synthesis is reduced in tau transgenic mice. Immunohistochemical staining of puromycin in non-transgenic (Non) and rTg4510 (Tg) at 5mo (a, b) and 7mo (c, d). e Quantification of panels a-d shows that puromycin signal was significantly reduced in $7 \mathrm{mo}$ Tg compared to control. Data analyzed by two-way ANOVA with Tukey's multiple comparisons test. $* p<0.05$ in comparisons outlined, $\&=0.071$ in comparison to $5 \mathrm{mo} \mathrm{Tg}$ at $5 \mathrm{mo}$ (Online Resource 5). However, since Tg mice have plasticity and cognitive deficits at $5 \mathrm{mo}[1,2]$, we speculated that this time point may reflect tau-induced modifications in the types of proteins that are being synthesized rather than causing an overall decrease in the rates of protein synthesis.

To determine whether tau impairs the translation of select proteins, we suppressed tau expression in the TET/OFF $\mathrm{Tg}$ system featured in $\mathrm{rTg} 4510$ mice with a doxycycline diet for 5 weeks from 3.5 to $4.75 \mathrm{mo}$ (Fig. 2a). This paradigm of doxycycline treatment rescues cognitive dysfunction and other neuronal deficits [51]. Therefore, any changes detected in protein levels as a consequence of tau expression would identify proteins that participate in the earliest stages of the pathological process. Importantly, we could also rule out proteins that do not participate in cognitive alterations if their levels would not change. We coupled our in vivo SUnSET method with anti-puromycin immunoprecipitation to facilitate proteomic identification of newly synthesized proteins. By integrating this proteomic analysis with microarray measures of transcript levels (Fig. 2b), we sought to determine whether suppression of P301L tau expression rescues translation of select proteins. As expected and as previously reported, we confirm that the doxycycline treatment reduced tau levels by $\sim 50 \%$ (Fig. 2c-d). This enabled the examination of the relationship between tau expression and RNA translation during the window of reversible cognitive dysfunction present in this model [52].

To assess how the nascent proteome changed as a function of tau expression, we performed mass spectrometry analysis of nascent, puromycinylated proteins immunoprecipitated from cortical tissue of control and $\mathrm{Tg}$ mice treated with or without doxycycline feed. We first examined whether doxycycline treatment in non-transgenic mice had a functional effect on the cortical puromycinylated nascent proteome. Annotations identified by the Database for Annotation, Visualization and Integrated Discovery (DAVID) revealed no difference between Non mice with or without doxycycline treatment (Fig. 3a and Online Resource 2). This enabled the comparison between the nascent proteome of Tg mice with or without suppression of tau expression to non-transgenic mice. Strikingly, much of the puromycinylated nascent proteome varied due to tau expression and suppression (Fig. 3b). However, the total mass of puromycinylated proteins isolated from cortex was unchanged (Online Resource 6), validating the lack of statistically significant overall translation differences at this time point found by anti-puromycin immunostaining. Pathway analysis via DAVID of these protein groups showed similar variation in statistically significantly enriched annotation terms (Fig. 3c). We next heuristically grouped the annotations into six distinct categories to illustrate differences in the pathways represented by the puromycinylated nascent proteome when tau is overexpressed and then suppressed (Fig. 3d). 

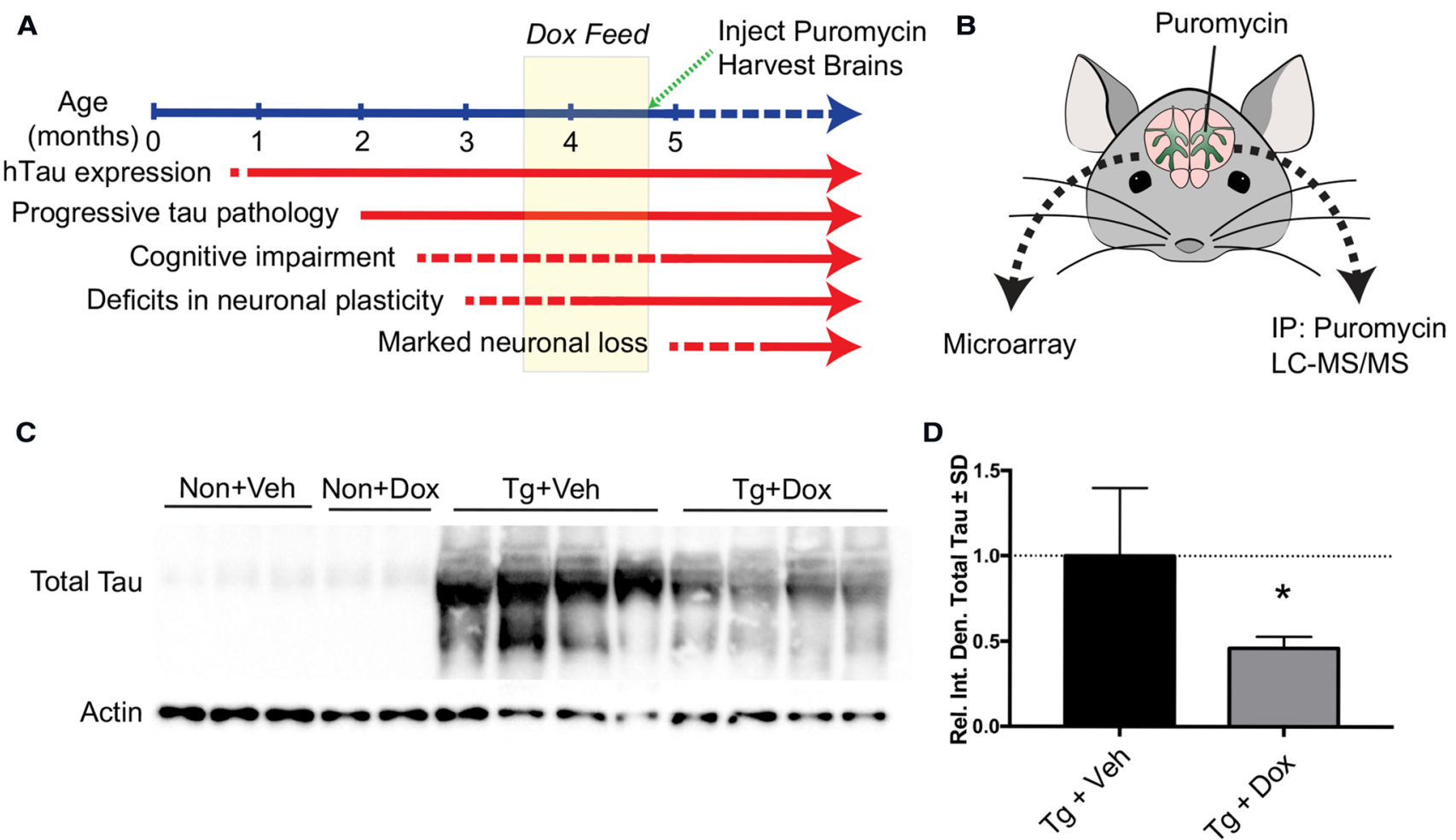

Fig. 2 Experimental design. a Timeline of Tg phenotype and strategy for inhibiting tau expression with doxycycline (dox). b Strategy for brain isolation and processing for microarrays or puromycin-based proteomics. c Representative immunoblot showing reduced total tau

As expected, the change in categories varied between tau expression and suppression. However, proteins involved in RNA translation and ribosomes were markedly reduced in tau-expressing mice, and doxycycline treatment rescued the synthesis of these proteins (Fig. 3d). These data indicate that tau expression reversibly altered the synthesis of translation machinery proteins in vivo, and it did so during the window where tau reduction rescues cognitive function.

To establish whether the changes in the translation of proteins identified from our proteomics approach were due to alterations in transcript levels, we profiled the transcriptome of Non and Tg mice treated with or without doxycycline using Clariom D microarrays. Differentially expressed genes (DEGs) were identified by two-way ANOVA ( $p \leq 0.01$; FDR $0.29 ; 1195 / 9537$ genes- $12.5 \%)$ and subsequently categorized by post hoc template matching (Methods: Fig. 4a-b; $91 \%$ of DEGs were assigned to one of these patterns). Three of the six templates were enriched above the levels of random chance and were considered as statistically significant patterns of differential expression (Fig. 4c, Online Resource 3). Pattern 1, "Rescued by Tau Reduction", corresponded to 406 transcripts $(\sim 4 \%)$ rescued in transgenic mice given doxycycline (Fig. 4d). Pattern 2, "Resistant to Tau Reduction", identified $106(\sim 1 \%)$ transcripts that increased in tau
(H150 antibody) levels after dox treatment compared to vehicle (veh) feed. d Quantification of $\mathbf{c}$ showing a 54\% reduction in tau signal after doxycycline treatment analyzed by two-tailed, unpaired $t$ test. $* p<0.05$

transgenic mice but unaffected by doxycycline treatment (Fig. 4e). Lastly, Pattern 3 established 333 transcripts ( 3\%) that were primarily affected by doxycycline treatment and not tau over-expression or suppression (Fig. 4f). Since this paradigm of doxycycline treatment rescues cognitive defects in Tg mice [51], these patterns suggest that we identified genes that mediate tau-driven cognitive impairment (Pattern 1), are not involved in cognitive rescue (Pattern 2), or that could be affected by doxycycline treatment paradigms (35d) used in this and other TET-dependent studies (Pattern 3). However, transcripts coding for proteins involved in translation were unchanged and were not categorized into any pattern of differential expression (Fig. 4g). Therefore, transcription was not a direct contributing factor to changes in the translation of ribosomal proteins, initiation factors, and other mediators of protein synthesis (Fig. 3d).

To define a mechanism by which tau exerts these changes, we focused on an innate process of translational regulation that is driven by the ribosomal protein S6. S6 is involved in regulating translation initiation and in facilitating the translation of ribosomal proteins, elongation factors, and initiation factors that contain a $5^{\prime}$ terminal oligopyrimidine (5'TOP) mRNA motif [24, 40, 49, 62]. These 5'TOP mRNAs are recognized by phosphorylated 
A

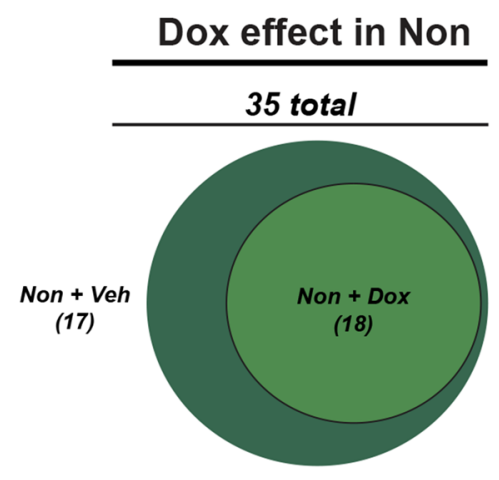

B
C

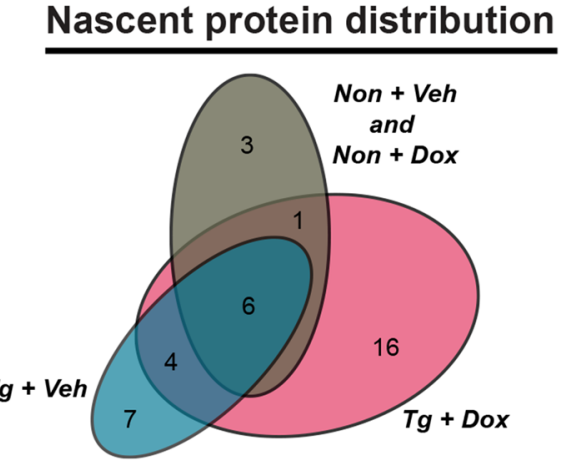

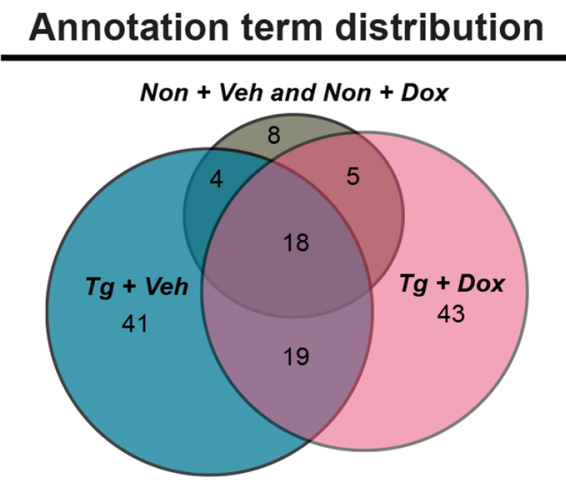

D

GO Combined Class
Translation and ribosomal
Metabolic and biosynthetic process
Immunological and inflammatory response
Cytoskeletal and structural
Protein trafficking, regulation, activity
Intra- and inter-cellular activity

Fig. 3 Pathological tau shifts the nascent proteomic profile. a Puromycinylated proteins were isolated by immunoprecipitation and analyzed using LC-MS/MS. a Doxycycline did not change the annotation profile in Non mice. b Venn diagram showing unique proteins identified by proteomics distributed between groups. $\mathbf{c}$ Venn diagram showing the distribution of annotation pathways representing pro-

S6 to accommodate ribosomal engagement and subsequent translation [18, 37]. In addition, we previously identified that pathological tau associates with S6 in AD [34]. Therefore, tau-mediated impairment of S6 activity could at least partly explain why proteins involved in translation were reduced in Tg mice (Fig. 3d).

To investigate the impact of tau on S6, we modulated human wild-type 0N4R tau expression in iHEK tau cells and measured changes in active (pS6) and total S6 levels. iHEK-tau cells are a tetracycline-inducible cell line that stably expresses WT human tau [4]. As expected, tetracycline treatment progressively increased PHF1 and total tau levels compared to no tetracycline controls (Fig. 5a, b). These time points also correlate to protein synthesis impairments as measured by puromycin incorporation in this cell line as previously shown [36]. Compared to no-tetracycline controls the ratio of S6 phosphorylation to total protein was unchanged at $24 \mathrm{~h}$ of tau expression, but at $96 \mathrm{~h}$ the ratio was reduced by $\sim 80 \%$ (Fig. $5 \mathrm{c}$ ). Following $24 \mathrm{~h}$ tetracycline washout and subsequent decrease of tau level, pS6 to total S6 levels were rescued by $30 \%$ and approximately doubled the levels found at $96 \mathrm{~h}$ alone. This rescue of S6 phosphorylation

\begin{tabular}{|c|c|c|c|}
\hline Non & Tg+Veh & Tg+Dox & $\begin{array}{r}\text { Enrichment } \\
\text { Ratio to Non }\end{array}$ \\
\hline 1.0 & 0.0 & 1.5 & 0.0 \\
\hline 1.0 & 0.5 & 3.5 & 0.5 \\
\hline 1.0 & 0.9 & 0.7 & 1.0 \\
\hline 1.0 & 1.5 & 5.5 & 5.0 \\
\hline 1.0 & 16.0 & 13.0 & 10.0 \\
\hline 1.0 & 11.5 & 9.5 & 16.0 \\
\hline
\end{tabular}

teins found in each group. d Categorized annotation terms identified in each group, separated by function and quantified as a ratio of the number of annotations present in Non. Ribosomal machinery and translation-related proteins were markedly reduced by tau expression and rescued with doxycycline treatment

suggested a potentially novel toxic gain-of-function where tau may preclude $\mathrm{S} 6$ phosphorylation.

To establish whether these effects occurred during neurodegenerative processes, particularly conditions where tau is not overexpressed, we measured the RNA and protein levels of translation-related, 5'TOP mRNAs in human AD brains grouped by mini-mental state exam (MMSE) scores. While early AD (MMSE > 20-25) samples had no significant increases in relative transcript levels of four candidate 5'TOP transcripts (RPS6, EIF3E, RPL28, and EIF2S1) compared to control, late AD (MMSE $\leq 20)$ samples exhibited fivefold or greater increases relative to control in all candidate transcript levels (Fig. 6a). Two transcripts were also significantly increased in late $\mathrm{AD}$ samples compared to early AD (RPS6 and EIF2S1). To assess whether these major differences in transcription evident in early and late $A D$ brain samples, we compared changes in 5'TOP-coded protein levels in the corresponding early and late AD samples (Fig. 6b). The levels of active pS6 and total S6 were both markedly reduced (by approximately 50\%; $p<0.01$ ) in late $\mathrm{AD}$ samples compared to control, with the ratio of $\mathrm{pS} 6 / \mathrm{S} 6$ trending toward a significant reduction $(p=0.092)$. Similarly, 5'TOP 
A

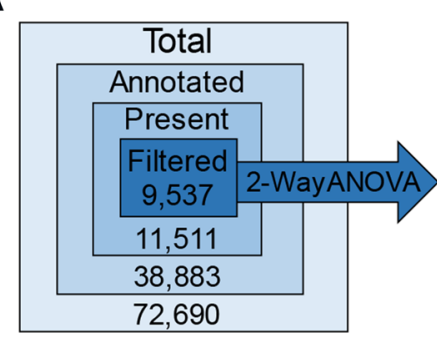

B

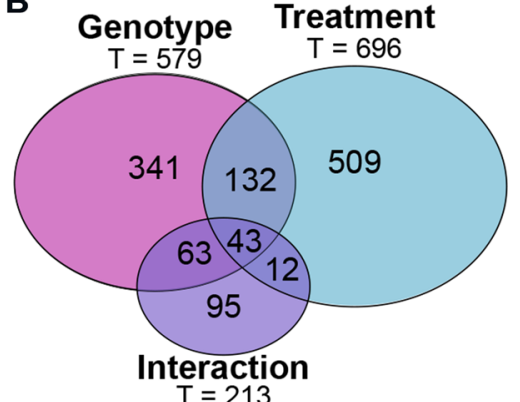

C

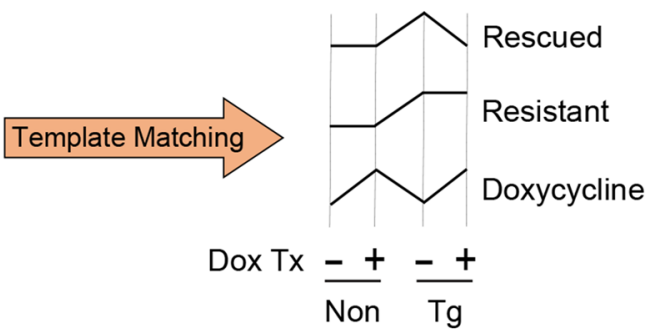

D

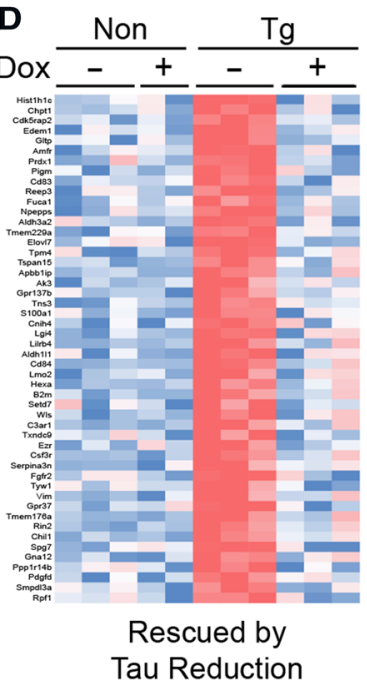

Fig. 4 Pathological tau and doxycycline modify transcriptomic profiles into three distinct patterns, but ribosomal profiles do not change. a Filtering strategy of microarray results to identify genes and patterns of differential expression. b Venn diagram showing the distribution of transcripts identified as significant by two-way ANOVA $(p \leq 0.01)$ across effects of genotype, treatment, and the interaction
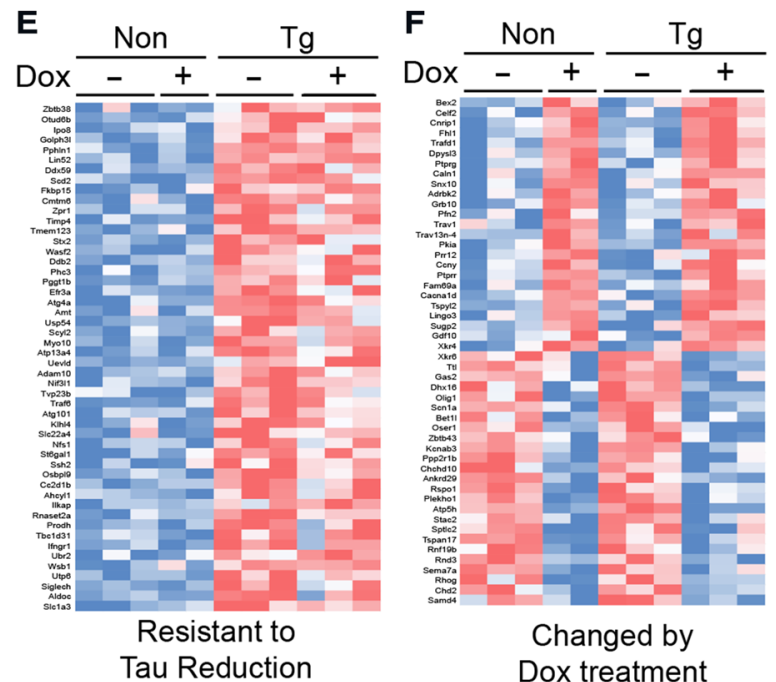
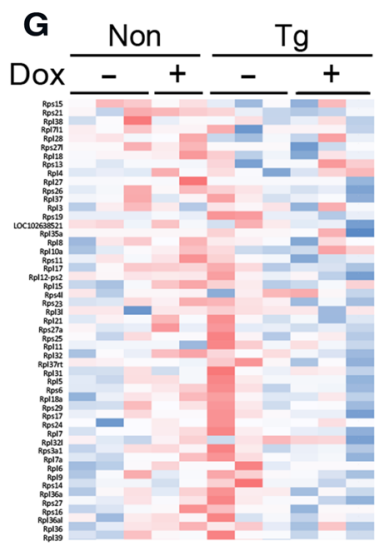

Ribosomal machinery or translation-related transcripts

Z-score

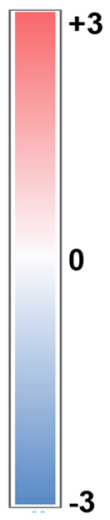

A

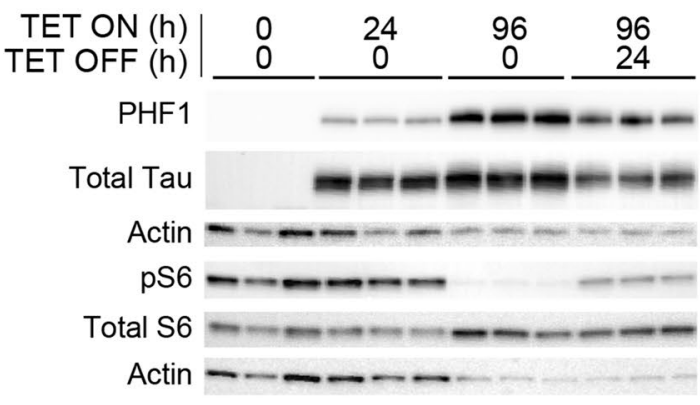

B

\section{PHF1 / Total Tau}

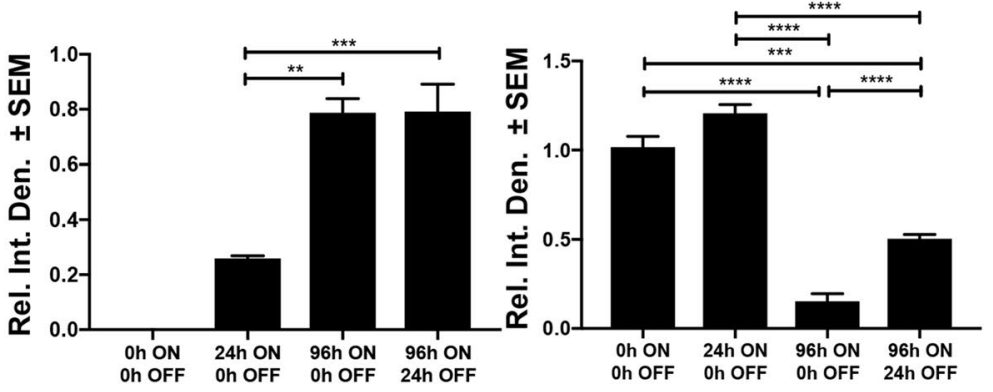

Fig. 5 Tau expression reduces pS6 in immortalized human cells. a Representative immunoblot showing the effects of tau expression in tetracycline-inducible iHEK-Tau cells. Tau was expressed for 24 or $96 \mathrm{~h}(\mathrm{ON})$ and either harvested immediately or following $24 \mathrm{~h}$ tetra- between both variables. c Differential expression patterns enriched beyond the levels expected by random chance. d-f Heat maps representing intensities $\left(\log _{2}\right)$ of transcripts matched to statistically significantly patterns of differential expression. $\mathbf{g}$ Heat map representing intensities $\left(\log _{2}\right)$ of transcripts coding for proteins related to translation including ribosomal proteins. cycline wash-out (OFF). Quantification of the ratio of PHF1 to total tau (H150 antibody) levels (b) or pS6 to total S6 levels (c). Data analyzed by two-way ANOVA with Tukey's multiple comparisons test. $* * p<0.01, * * * p<0.001, * * * * p<0.0001$ 
A

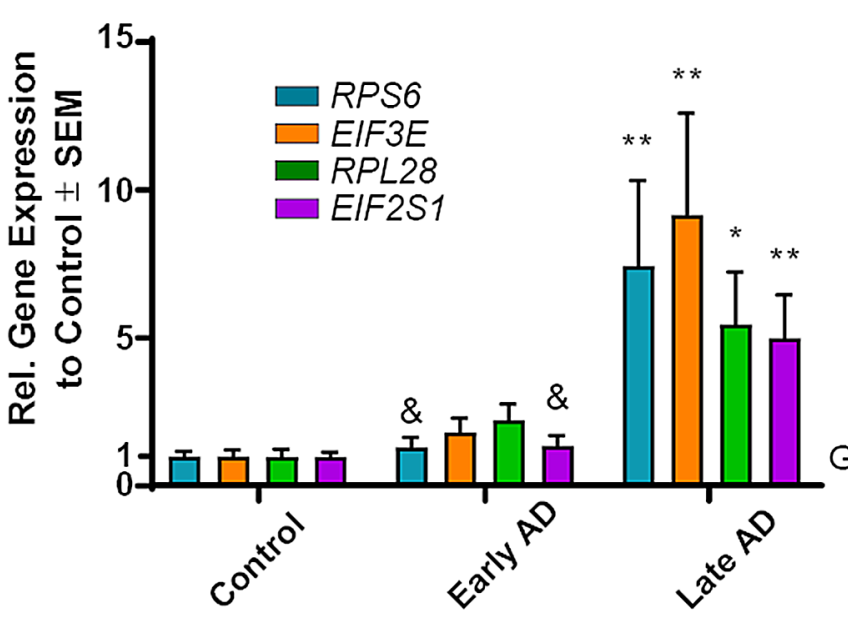

D

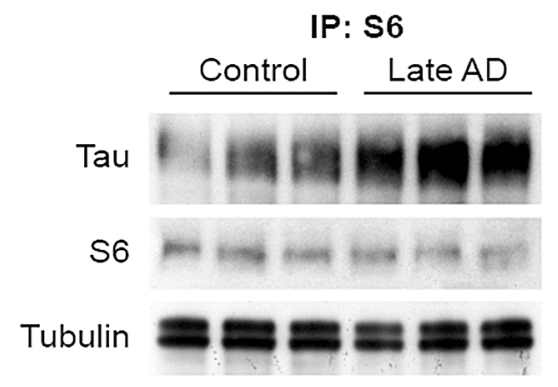

E

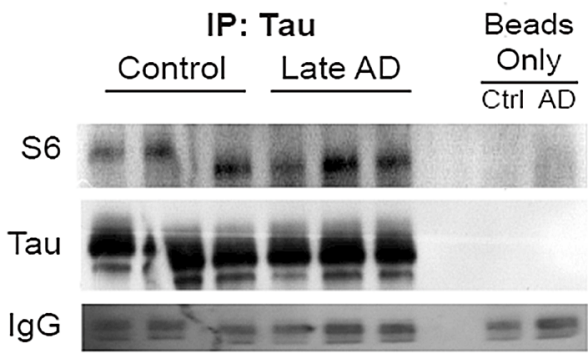

elF3E the
B

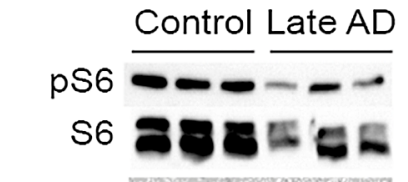

RPL28

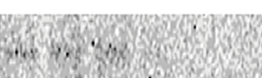

PHF1

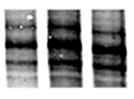

GAPDH

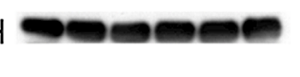

$\mathbf{F}$

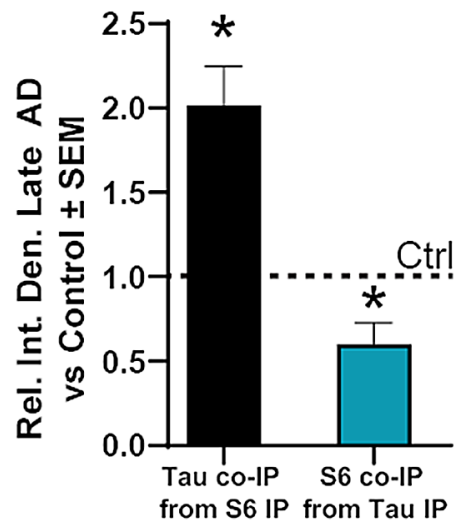

Fig. 6 S6 exhibits impaired function and increased association with tau in AD. a RT-Q-PCR of human brain RNA isolate reveals no change in 5'TOP mRNA levels in early AD but substantial increases in late AD compared to control (Kruskal-Wallis with Dunn's multiple comparison test, $n=9-10$ ). b Representative immunoblot showing reduced $\mathrm{pS6}$, total $\mathrm{S6}$, and 5'TOP protein synthesis in late $\mathrm{AD}$ brains with PHF1 signal compared to control (one-way ANOVA with Tukey's multiple comparison's test, $n=6-12$ ). c Quantification of pS6

rpL28 and eIF3E were also significantly reduced $(\sim 55 \%$ at $p<0.001$ and $75 \%$ at $p<0.05$, respectively) (Fig. 6c) indicating that the protein synthesis of these translation-related transcripts was impaired as observed in tau transgenic mice (Fig. 3d).

Since pathological tau species associate with S6 in AD [33], and pS6 promotes translation of 5'TOP mRNAs [24], we speculated that $\mathrm{AD}$ brains contained tau-S6 complexes. We found that $\mathrm{S} 6$ and tau co-immunoprecipitated together in both control and late AD brains (Fig. 6d, e). Quantification of this signal showed a nearly twofold increase of tau associated with $\mathrm{S} 6$ in late $\mathrm{AD}$ relative to control, suggesting a potentially stronger interaction of S6 with disease-associated tau (Fig. 6f). Quantification of the S6 pool associated and 5'TOP protein levels. S6 (d) and total tau (Tau 5 antibody, e) coimmunoprecipitate from human $\mathrm{AD}$ and control brain lysate regardless of host protein. Tissue lysate incubated without antibodies were used in the beads only lanes. IgG bands confirm the use of beads in all sample preparations, $(n=3)$. f Quantification of co-immunoprecipitation as a ratio to control. Each protein independently tested to control protein levels with two-tailed, unpaired Student's $t$ test. ${ }^{*} p<0.05$, ${ }^{* *} p<0.01, * * * p<0.001, * * * * p<0.0001,{ }^{\&} p<0.10$

with tau revealed a $60 \%$ reduction in S6 compared to control, reflective of the decrease in total $\mathrm{S} 6$ found in late $\mathrm{AD}$ brain (Fig. 6b). These results show that tau-S6 complexes correlate with a reduced S6 function and consequently less ribosomal machinery in $\mathrm{AD}$. Overall, these data suggest that tau associates with S6 and shifts the types of transcripts that are preferentially translated, eventually amounting to impairment in global translation. 


\section{Discussion}

Increasing evidence suggests that impaired translation contributes to the pathogenesis of neurodegenerative diseases like AD and ALS [29]. Over the last several decades, tau has been implicated as a modifier of a growing number of cellular processes across tauopathies, but the mechanisms of taumediated toxicity remain unclear [56]. Here, we expanded our previous findings on tau-mediated impairments in translation to show a potential mechanism describing how pathological tau modulates the selectivity and activity of ribosomes. We coupled transcriptomics and nascent proteomics with validation in vitro and in human brain samples from patients with $\mathrm{AD}$ to discern that tau reduces the activity of ribosomal protein S6, a crucial regulator of translation. In this context, we propose that tau pathology impacts translation, unveiling new prospects for therapeutic intervention. Since impaired translation may impact many pathways in tauopathies such as synaptic plasticity, cellular metabolism, and memory formation, tau-mediated impairments in translation may explain a mechanism by which tau directly promotes disease.

We establish that translation is shifted in early stages of progressive tau pathology in the $\mathrm{rTg} 4510$ mouse model of tauopathy (Fig. 1). We identified reduced protein synthesis at a later disease stage (7mo). Importantly, our in vivo SUnSET method validates a previous study that showed reduced ${ }^{35} \mathrm{~S}$-methionine incorporation as a proxy of global translation at $7 \mathrm{mo}$ in $\mathrm{rTg} 4510$ [47]. Since no statistically significant decrease in protein synthesis has been determined by any study including our own at the cognitive-reversal window in $\mathrm{rTg} 4510$ mice, we hypothesized this earlier time point may reflect a turning point in translation that correlates with disease progression [51]. We, therefore, sought to couple transcriptomics and proteomic profiles of newly synthesized proteins, or nascent proteomics.

Parallel analyses of the transcriptome and nascent proteome revealed that, as expected, this early time point had marked differences in gene expression as a product of tau accumulation (Figs. 3, 4). As expected based on our previous results [34], synthesis of many proteins was suppressed by tau expression; however, we also identified many proteins that were increased as a consequence of tau expression, which corroborates results that were recently described [41]. Strikingly, transcript levels coding for ribosomal genes were unchanged while the protein levels were rescued by tau suppression, supporting the hypothesis that a shift in translation occurs during the window where doxycycline treatment rescues cognitive impairment in these mice. The results mirror past studies that found opposing effects on ribosomal gene transcript and protein levels in brain samples from patients with tauopathies [17, 28]. Moreover, these findings are not surprising considering the recruitment of tau-ribosome complexes in stress granules with TIA1 [7, 59] Based on our microarray analysis, we also isolated a pronounced doxycycline effect (Fig. 4f). Doxycycline treatment has been previously shown to alter physiological function and translation in vivo [36]. Interestingly, the affected genes corresponded primarily to inflammatory proteins. Since this potentially confounds the direct comparison of translation and multi-omics studies in our rTg4510 experiments, we sought to validate these findings in vitro. Importantly, to avoid potential issues with models of overexpression, we complemented our studies using human AD brain samples, which do not have tau overexpression [33].

While the reduction in total polysome levels [31], ribosome translational efficiency [17], and 5'TOP protein levels [17] have been well established in $\mathrm{AD}$, no study has presented evidence mechanistically linking decreases in ribosome function with tau. We previously found that toxic tau species co-localized with S6 in AD brain [34], supporting other findings that tau associates with ribosomes [28, 40, $41,43-45,58]$. Since S6 is a critical regulator of ribosomal biogenesis and activity, we investigated how the activity of S6 changes in tauopathic conditions in vitro and in human brains. We measured changes in S6 phosphorylation at Ser240/244 because this site is a marker for its localization into the somatodendritic domain, which is also where tau mislocalizes in disease $[9,18,45,46,55]$. The levels of pS6 were reversibly affected by tau levels in vitro (Fig. 5) and inversely proportional to PHF1 tau in AD brains (Fig. 6). This reduction in the functional pool of S6 also correlated with a fivefold or greater increase in transcript levels but a halving of protein levels of 5'TOP translation-related genes suggesting an impairment in 5'TOP transcript translation. We also identified a twofold increase in the amount of tau associated with S6 in late AD brains, suggesting that this decrease in $\mathrm{S} 6$ function is related to the direct association with tau in humans. Moreover, since reductions in functional pools of S6 correlate to reductions in LTP [48] and substantial dendritic atrophy [56], the tau-S6 complex may be pathologically relevant in the development or progression of cognitive dysfunction and merits further investigation. Overall, these data strongly suggest a gain of toxic function where tau associates with and prevents the phosphorylation of S6, thereby altering the selectivity and translational capability of the ribosome (Fig. 7).

While we present a mechanistic link between ribosomal dysfunction and tau pathology in $\mathrm{AD}$, other potential direct and indirect factors may contribute to impair translation. For example, oxidative damage is linked to decreased ribosomal integrity and protein translation rates [21, 60], and the effect of tau on increasing oxidative stress is well established. Furthermore, toxic amyloid beta oligomers also induce rRNA damage and translational impairment [31]. 


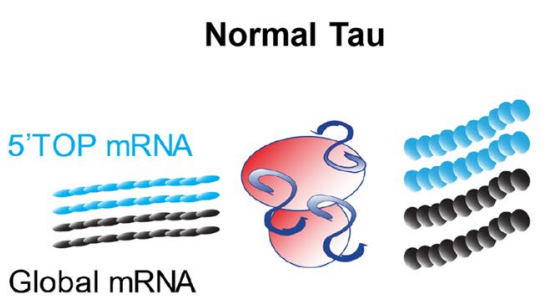

Fig. 7 Schematic representation of overall conclusions. Tau normally associates with ribosomes and facilitates translation between global transcripts and 5'TOP mRNA sequences. In disease conditions, pathological tau species reduce the efficiency of translation and pre-

Importantly, many other factors implicated in the pathogenesis of tauopathies alter S6 activity, such as S6K1 and S6K2. Interestingly, one study linked S6 kinase activity to AD phenotype in 5xFAD mice, where reducing S6 kinase activity conferred cognitive benefits [12]. This apparent discrepancy with our experiments may be due to the ability for S6 kinases to phosphorylate both active sites on S6 [9], which in turn have different and inconclusively determined effects in regulating S6 function and translation. Moreover, translation of 5'TOP transcripts is independent to S6K1 activity, suggesting that more potential factors regulate the function of S6 to regulate ribosomal function [58]. Ultimately, more sensitive measures to assess active translation are needed to more thoroughly elucidate the direct and indirect methods by which tau mediates translational impairments in disease.

Acknowledgements We thank Dr. Peter Nelson, Ela Patel, and Sonya Anderson, the University of Kentucky Alzheimer's Disease Center (UK-ADC). We also thank Dr. Steven Estus and James Simpson for their technical support, Dr. Peter Davies for his generous contribution of the PHF1 antibody, and Dr. Chad Dickey for the inspiration for this work and developing and sharing the iHEK cell lines and the tau plasmids.

Author contributions SAK, SEM, JFA designed the experiments and conceptualized the paper. BEW and JPA completed the immunostaining. $\mathrm{HZ}$ and $\mathrm{JC}$ conducted the proteomic experiments and analysis. SAK and EMB analyzed microarray data. SAK, MJH, SEM, GKN, and EAC completed biochemical experiments and SAK and MJH subsequent analysis. SAK and JFA wrote the manuscript.

Funding This work was funded by NIH R01NS077284 (HZ) and Alzheimer's Association NIRG-14-322441, Department of Defense AZ140097, NIH/NIMHD L32 MD009205-01, NIH 1R21NS093440, NIH/NINDS 1R01 NS091329-01 (JFA). Brain samples were obtained from the University of Kentucky ADC, which is supported by NIH/ NIA P30 AG028383. The University of Kentucky Proteomics Core is supported by NIH/NIGMS P20GM103486, NIH/NCI P30CA177558, NIH S10RR029127.

Open Access This article is distributed under the terms of the Creative Commons Attribution 4.0 International License (http://creativeco mmons.org/licenses/by/4.0/), which permits unrestricted use, distribution, and reproduction in any medium, provided you give appropriate

\section{Pathological Tau}

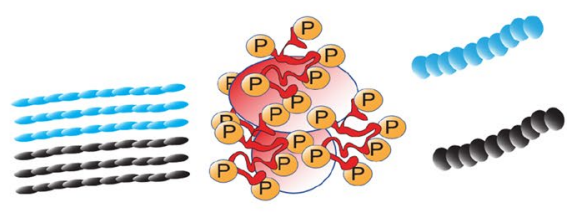

vent rpS6 phosphorylation at S240/S244. As rpS6 activity decreases, translation of 5'TOP mRNAs coding for ribosomal and translational machinery is reduced, impairing global translation

credit to the original author(s) and the source, provide a link to the Creative Commons license, and indicate if changes were made.

\section{References}

1. Abisambra J, Jinwal UK, Miyata Y, Rogers J, Blair L, Li X et al (2013) Allosteric heat shock protein 70 inhibitors rapidly rescue synaptic plasticity deficits by reducing aberrant tau. Biol Psychiatry 74:367-374. https://doi.org/10.1016/j.biopsych.2013.02.027

2. Abisambra JF, Blair LJ, Hill SE, Jones JR, Kraft C, Rogers J et al (2010) Phosphorylation dynamics regulate Hsp27-mediated rescue of neuronal plasticity deficits in tau transgenic mice. J Neurosci 30:15374-15382. https://doi.org/10.1523/JNEUR OSCI.3155-10.2010

3. Abisambra JF, Fiorelli T, Padmanabhan J, Neame P, Wefes I, Potter H (2010) LDLR expression and localization are altered in mouse and human cell culture models of Alzheimer's disease. PLoS One 5:e8556. https://doi.org/10.1371/journal.pone.0008556

4. Abisambra JF, Jinwal UK, Blair LJ, O'Leary JC 3rd, Li Q, Brady $S$ et al (2013) Tau accumulation activates the unfolded protein response by impairing endoplasmic reticulum-associated degradation. J Neurosci 33:9498-9507. https://doi.org/10.1523/JNEUR OSCI.5397-12.2013

5. Abisambra JF, Jinwal UK, Suntharalingam A, Arulselvam K, Brady S, Cockman M (2012) DnaJA1 antagonizes constitutive Hsp70-mediated stabilization of tau. J Mol Biol 421:653-661. https://doi.org/10.1016/j.jmb.2012.02.003

6. Alsiraj Y, Thatcher SE, Blalock E, Fleenor B, Daugherty A, Cassis LA (2018) Sex chromosome complement defines diffuse versus focal angiotensin II-induced aortic pathology. Arterioscler Thromb Vasc Biol 38:143-153. https://doi.org/10.1161/ATVBA HA.117.310035

7. Apicco DJ, Ash PEA, Maziuk B, LeBlang C, Medalla M, Al Abdullatif A et al (2018) Reducing the RNA binding protein TIA1 protects against tau-mediated neurodegeneration in vivo. Nat Neurosci 21:72-80. https://doi.org/10.1038/s41593-017-0022-z

8. Barna M (2015) The ribosome prophecy. Nat Rev Mol Cell Biol 16:268. https://doi.org/10.1038/nrm3993

9. Biever A, Valjent E, Puighermanal E (2015) Ribosomal protein S6 phosphorylation in the nervous system: from regulation to function. Front Mol Neurosci 8:75. https://doi.org/10.3389/fnmol .2015 .00075

10. Blalock EM, Korrect GS, Stromberg AJ, Erickson DR (2012) Gene expression analysis of urine sediment: evaluation for potential noninvasive markers of interstitial cystitis/bladder 
pain syndrome. J Urol 187:725-732. https://doi.org/10.1016/j. juro.2011.09.142

11. Bolstad BM, Irizarry RA, Astrand M, Speed TP (2003) A comparison of normalization methods for high density oligonucleotide array data based on variance and bias. Bioinformatics 19:185-193

12. Caccamo A, Magrì A, Medina DX, Wisely EV, López-Aranda MF, Silva AJ (2013) mTOR regulates tau phosphorylation and degradation: implications for Alzheimer's disease and other tauopathies. Aging Cell 45:54. https://doi.org/10.1111/acel.12057

13. Dhar SK, Zhang J, Gal J, Xu Y, Miao L, Lynn BC (2014) FUsed in sarcoma is a novel regulator of manganese superoxide dismutase gene transcription. Antioxid Redox Signal 20:1550-1566. https ://doi.org/10.1089/ars.2012.4984

14. Ding Q, Markesbery WR, Chen Q, Li F, Keller JN (2005) Ribosome dysfunction is an early event in Alzheimer's disease. J Neurosci 25:9171-9175. https://doi.org/10.1523/JNEUR OSCI.3040-05.2005

15. Duvarci S, Nader K, LeDoux JE (2008) De novo mRNA synthesis is required for both consolidation and reconsolidation of fear memories in the amygdala. Learn Mem 15:747-755. https ://doi.org/10.1101/lm.1027208

16. Gant JC, Blalock EM, Chen KC, Kadish I, Thibault O, Porter $\mathrm{NM}$ et al (2018) FK506-binding protein 12.6/1b, a negative regulator of $[\mathrm{Ca}(2+)]$, rescues memory and restores genomic regulation in the hippocampus of aging rats. J Neurosci 38:10301041. https://doi.org/10.1523/JNEUROSCI.2234-17.2017

17. Garcia-Esparcia P, Sideris-Lampretsas G, Hernandez-Ortega K, Grau-Rivera O, Sklaviadis T, Gelpi E et al (2017) Altered mechanisms of protein synthesis in frontal cortex in Alzheimer disease and a mouse model. Am J Neurodegener Dis 6:15-25

18. Gobert D, Topolnik L, Azzi M, Huang L, Badeaux F, Desgroseillers L et al (2008) Forskolin induction of late-LTP and upregulation of 5'TOP mRNAs translation via mTOR, ERK, and PI3 K in hippocampal pyramidal cells. J Neurochem 106:11601174. https://doi.org/10.1111/j.1471-4159.2008.05470.x

19. Gunawardana CG, Mehrabian M, Wang X, Mueller I, Lubambo IB, Jonkman JE et al (2015) The human tau interactome: binding to the ribonucleoproteome, and impaired binding of the proline-to-leucine mutant at position 301 (P301L) to chaperones and the proteasome. Mol Cell Proteom 14:3000-3014. https:// doi.org/10.1074/mcp.M115.050724

20. Harding HP, Zhang Y, Ron D (1999) Protein translation and folding are coupled by an endoplasmic-reticulum-resident kinase. Nature 397:271-274. https://doi.org/10.1038/16729

21. Hernandez-Ortega K, Garcia-Esparcia P, Gil L, Lucas JJ, Ferrer I (2016) Altered machinery of protein synthesis in Alzheimer's: from the nucleolus to the ribosome. Brain Pathol 26:593-605. https://doi.org/10.1111/bpa.12335

22. Hoeffer CA, Cowansage KK, Arnold EC, Banko JL, Moerke NJ, Rodriguez $\mathrm{R}$ et al (2011) Inhibition of the interactions between eukaryotic initiation factors $4 \mathrm{E}$ and $4 \mathrm{G}$ impairs long-term associative memory consolidation but not reconsolidation. Proc Natl Acad Sci USA 108:3383-3388. https://doi.org/10.1073/ pnas. 1013063108

23. da Huang W, Sherman BT, Lempicki RA (2009) Systematic and integrative analysis of large gene lists using DAVID bioinformatics resources. Nat Protoc 4:44-57. https://doi.org/10.1038/ nprot.2008.211

24. Iadevaia V, Caldarola S, Tino E, Amaldi F, Loreni F (2008) All translation elongation factors and the e, f, and h subunits of translation initiation factor 3 are encoded by 5 '-terminal oligopyrimidine (TOP) mRNAs. RNA 14:1730-1736. https://doi. org/10.1261/rna.1037108

25. Iseki E, Matsumura T, Marui W, Hino H, Odawara T, Sugiyama $\mathrm{N}$ et al (2001) Familial frontotemporal dementia and parkinsonism with a novel $\mathrm{N} 296 \mathrm{H}$ mutation in exon 10 of the tau gene and a widespread tau accumulation in the glial cells. Acta Neuropathol 102:285-292

26. Jinwal UK, Koren J, O'Leary JC, Jones JR, Abisambra JF, Dickey CA (2010) Hsp70 ATPase modulators as therapeutics for Alzheimer's and other neurodegenerative diseases. Mol Cell Pharmacol 2:43-46

27. Kadish I, Thibault O, Blalock EM, Chen KC, Gant JC, Porter $\mathrm{NM}$ et al (2009) Hippocampal and cognitive aging across the lifespan: a bioenergetic shift precedes and increased cholesterol trafficking parallels memory impairment. J Neurosci 29:18051816. https://doi.org/10.1523/JNEUROSCI.4599-08.2009

28. Langstrom NS, Anderson JP, Lindroos HG, Winblad B, Wallace WC (1989) Alzheimer's disease-associated reduction of polysomal mRNA translation. Brain Res Mol Brain Res 5:259-269

29. Lehmkuhl EM, Zarnescu DC (2018) Lost in translation: evidence for protein synthesis deficits in ALS/FTD and related neurodegenerative diseases. Adv Neurobiol 20:283-301. https ://doi.org/10.1007/978-3-319-89689-2_11

30. Maimaiti S, Anderson KL, DeMoll C, Brewer LD, Rauh BA, Gant JC et al (2016) Intranasal insulin improves age-related cognitive deficits and reverses electrophysiological correlates of brain aging. J Gerontol Ser A Biol Sci Med Sci 71:30-39. https://doi. org/10.1093/gerona/glu314

31. Maina MB, Bailey LJ, Doherty AJ, Serpell LC (2018) The involvement of abeta42 and tau in nucleolar and protein synthesis machinery dysfunction. Front Cell Neurosci 12:220. https://doi. org/10.3389/fncel.2018.00220

32. Mann DM, Neary D, Yates PO, Lincoln J, Snowden JS, Stanworth $P$ (1981) Alterations in protein synthetic capability of nerve cells in Alzheimer's disease. J Neurol Neurosurg Psychiatry 44:97-102

33. Meier S, Bell M, Lyons DN, Ingram A, Chen J, Gensel JC et al (2015) Identification of novel tau interactions with endoplasmic reticulum proteins in alzheimer's disease brain. J Alzheimer's Dis 48:687-702. https://doi.org/10.3233/JAD-150298

34. Meier S, Bell M, Lyons DN, Rodriguez-Rivera J, Ingram A, Fontaine SN et al (2016) Pathological tau promotes neuronal damage by impairing ribosomal function and decreasing protein synthesis. J Neurosci 36:1001-1007. https://doi.org/10.1523/JNEUR OSCI.3029-15.2016

35. Moreno JA, Radford H, Peretti D, Steinert JR, Verity N, Martin MG et al (2012) Sustained translational repression by eIF2alpha-P mediates prion neurodegeneration. Nature 485:507-511. https:// doi.org/10.1038/nature 11058

36. Moullan M, Ahossi V, Zwetyenga N (2016) Stevens-Johnson syndrome and toxic epidermal necrolysis (SJSTEN) related to insecticide: second case in the literature and potential implications. Revue de stomatologie, de chirurgie maxillo-faciale et de chirurgie orale 117:176-182. https://doi.org/10.1016/j.revst o.2016.04.003

37. Nandagopal N, Roux PP (2015) Regulation of global and specific mRNA translation by the mTOR signaling pathway. Translation (Austin) 3:e983402. https://doi.org/10.4161/21690 731.2014.983402

38. Nelson PT, Marton L, Saper CB (1993) Alz-50 immunohistochemistry in the normal sheep striatum: a light and electron microscope study. Brain Res 600:285-297

39. Nelson PT, Saper CB (1995) Ultrastructure of neurofibrillary tangles in the cerebral cortex of sheep. Neurobiol Aging 16:315-323

40. Nygard O, Nilsson L (1990) Kinetic determination of the effects of ADP-ribosylation on the interaction of eukaryotic elongation factor 2 with ribosomes. J Biol Chem 265:6030-6034

41. Pace MC, Xu G, Fromholt S, Howard J, Crosby K, Giasson BI et al (2018) Changes in proteome solubility indicate widespread proteostatic disruption in mouse models of neurodegenerative disease. Acta Neuropathol 136:919-938. https://doi.org/10.1007/ s00401-018-1895-y 
42. Papasozomenos SC (1989) Tau protein immunoreactivity in dementia of the Alzheimer type. I. Morphology, evolution, distribution, and pathogenetic implications. Lab Investig 60:123-137

43. Papasozomenos SC, Binder LI (1987) Phosphorylation determines two distinct species of tau in the central nervous system. Cell Motil Cytoskelet 8:210-226. https://doi.org/10.1002/cm.97008 0303

44. Piao YS, Hayashi S, Wakabayashi K, Kakita A, Aida I et al (2002) Cerebellar cortical tau pathology in progressive supranuclear palsy and corticobasal degeneration. Acta Neuropathol 103:469 474. https://doi.org/10.1007/s00401-001-0488-2

45. Pirbhoy PS, Farris S, Steward O (2016) Synaptic activation of ribosomal protein S6 phosphorylation occurs locally in activated dendritic domains. Learn Mem 23:255-269. https://doi. org/10.1101/lm.041947.116

46. Pirbhoy PS, Farris S, Steward O (2017) Synaptically driven phosphorylation of ribosomal protein S6 is differentially regulated at active synapses versus dendrites and cell bodies by MAPK and PI3K/mTOR signaling pathways. Learn Mem 24:341-357. https ://doi.org/10.1101/lm.044974.117

47. Radford H, Moreno JA, Verity N, Halliday M, Mallucci GR (2015) PERK inhibition prevents tau-mediated neurodegeneration in a mouse model of frontotemporal dementia. Acta Neuropathol 130:633-642. https://doi.org/10.1007/s00401-015-1487-z

48. Ramsden M, Kotilinek L, Forster C, Paulson J, McGowan E, SantaCruz K et al (2005) Age-dependent neurofibrillary tangle formation, neuron loss, and memory impairment in a mouse model of human tauopathy (P301L). J Neurosci 25:10637-10647. https:// doi.org/10.1523/JNEUROSCI.3279-05.2005

49. Roux PP, Shahbazian D, Vu H, Holz MK, Cohen MS, Taunton $J$ et al (2007) RAS/ERK signaling promotes site-specific ribosomal protein S6 phosphorylation via RSK and stimulates capdependent translation. J Biol Chem 282:14056-14064. https://doi. org/10.1074/jbc.M700906200

50. Sajdel-Sulkowska EM, Marotta CA (1984) Alzheimer's disease brain: alterations in RNA levels and in a ribonuclease-inhibitor complex. Science 225:947-949

51. Santacruz K, Lewis J, Spires T, Paulson J, Kotilinek L, Ingelsson $\mathrm{M}$ et al (2005) Tau suppression in a neurodegenerative mouse model improves memory function. Science 309:476-481. https ://doi.org/10.1126/science.1113694

52. Schmidt EK, Clavarino G, Ceppi M, Pierre P (2009) SUnSET, a nonradioactive method to monitor protein synthesis. Nat Methods 6:275-277. https://doi.org/10.1038/nmeth.1314

53. Simsek D, Barna M (2017) An emerging role for the ribosome as a nexus for post-translational modifications. Curr Opin Cell Biol 45:92-101. https://doi.org/10.1016/j.ceb.2017.02.010

54. Simsek D, Tiu GC, Flynn RA, Byeon GW, Leppek K, Xu $\mathrm{AF}$ et al (2017) The mammalian ribo-interactome reveals ribosome functional diversity and heterogeneity. Cell 169(10511065):e1018. https://doi.org/10.1016/j.cell.2017.05.022

55. Slomnicki LP, Pietrzak M, Vashishta A, Jones J, Lynch N, Elliot $S$ et al (2016) Requirement of neuronal ribosome synthesis for growth and maintenance of the dendritic tree. J Biol Chem 291:5721-5739. https://doi.org/10.1074/jbc.M115.682161

56. Sotiropoulos I, Galas MC, Silva JM, Skoulakis E, Wegmann S, Maina MB et al (2017) Atypical, non-standard functions of the microtubule associated tau protein. Acta Neuropathol Commun 5:91. https://doi.org/10.1186/s40478-017-0489-6

57. Tanemura K, Murayama M, Akagi T, Hashikawa T, Tominaga T, Ichikawa $\mathrm{M}$ et al (2002) Neurodegeneration with tau accumulation in a transgenic mouse expressing V337M human tau. J Neurosci 22:133-141

58. Tang H, Hornstein E, Stolovich M, Levy G, Livingstone M, Templeton D et al (2001) Amino acid-induced translation of TOP mRNAs is fully dependent on phosphatidylinositol 3-kinase-mediated signaling, is partially inhibited by rapamycin, and is independent of S6K1 and rpS6 phosphorylation. Mol Cell Biol 21:8671-8683. https://doi.org/10.1128/ MCB.21.24.8671-8683.2001

59. Vanderweyde T, Apicco DJ, Youmans-Kidder K, Ash PE, Cook C, Lummertz da Rocha E et al (2016) Interaction of tau with the RNA-binding protein TIA1 regulates tau pathophysiology and toxicity. Cell Rep 15:1455-1466. https://doi.org/10.1016/j.celre p.2016.04.045

60. Willi J, Kupfer P, Evequoz D, Fernandez G, Katz A, Leumann C et al (2018) Oxidative stress damages rRNA inside the ribosome and differentially affects the catalytic center. Nucleic Acids Res 46:1945-1957. https://doi.org/10.1093/nar/gkx1308

61. Xue S, Barna M (2015) Cis-regulatory RNA elements that regulate specialized ribosome activity. RNA Biol 12:1083-1087. https ://doi.org/10.1080/15476286.2015.1085149

62. Yamashita R, Suzuki Y, Takeuchi N, Wakaguri H, Ueda T, Sugano $S$ et al (2008) Comprehensive detection of human terminal oligo-pyrimidine (TOP) genes and analysis of their characteristics. Nucleic Acids Res 36:3707-3715. https://doi.org/10.1093/ nar/gkn248

63. Zhai J, Strom AL, Kilty R, Venkatakrishnan P, White J, Everson WV, Smart EJ et al (2009) Proteomic characterization of lipid raft proteins in amyotrophic lateral sclerosis mouse spinal cord. FEBS J 276:3308-3323. https://doi.org/10.111 1/j.1742-4658.2009.07057.x

Publisher's Note Springer Nature remains neutral with regard to jurisdictional claims in published maps and institutional affiliations. 